\title{
IDIOPATHIC CAROTID JUGULAR FISTULA PRESENTING AS PULSATILE TINNITUS
}

\section{Emamikhah ${ }^{1}$, B. Zamani ${ }^{2}$, N. Mohebi ${ }^{1}$}

'Department of neurology-Rasool-e Akram hospital- Iran university of medical sciences- Tehran- Iran, Department of neurology, Tehran, Iran. 2Department of neurology-Firoozgar hospital- Iran university of medical sciences- Tehran- Iran., Department of neurology, Tehran, Iran.

\section{Background and Aims}

Carotid - jugular fistula (CJF), an abnormal connection between the carotid artery and jugular vein, etiologically is described as congenital or acquired. Congenital CJFs are less common. Leading causes for acquired CJFs are trauma and iatrogenic.

Other etiologic conditions have been presumed, but these rare cases mostly remain idiopathic.

CJFs of either etiology can present with different sign and symptoms such as pulsatile mass, palpable thrill, auscultable murmur or dilated veins, wide pulse pressure, low diastolic blood pressure and in severe cases with heart failure. Decreased cerebral arterial flow or increased venous congestion can lead to neurological sign and symptoms.

The gold standard diagnostic test remains digital subtraction angiography (DSA), but duplex ultrasound (DU) is an easy sensitive method. Computed tomography angiography (CTA) \& magnetic resonance angiography (MRA) are other sensitive options.

\section{Methods:}

We present a case of idiopathic CJF diagnosed during work up for pulsatile tinnitus.

\section{Results:}

A 72 year old female presented with a pulsating tinnitus. Routine work up was unremarkable. Cervical DU revealed internalized external carotid artery (ECA).

\section{Conclusions:}

CJFs of non-traumatic non-iatrogenic cause should be considered in cases of neurologic symptoms of unknown etiology attributable to cerebral flow change, especially if regional or systemic circulatory symptoms are present simultaneously. Early detection by means of appropriate diagnostic tool and in time management prevents irreversible complications.
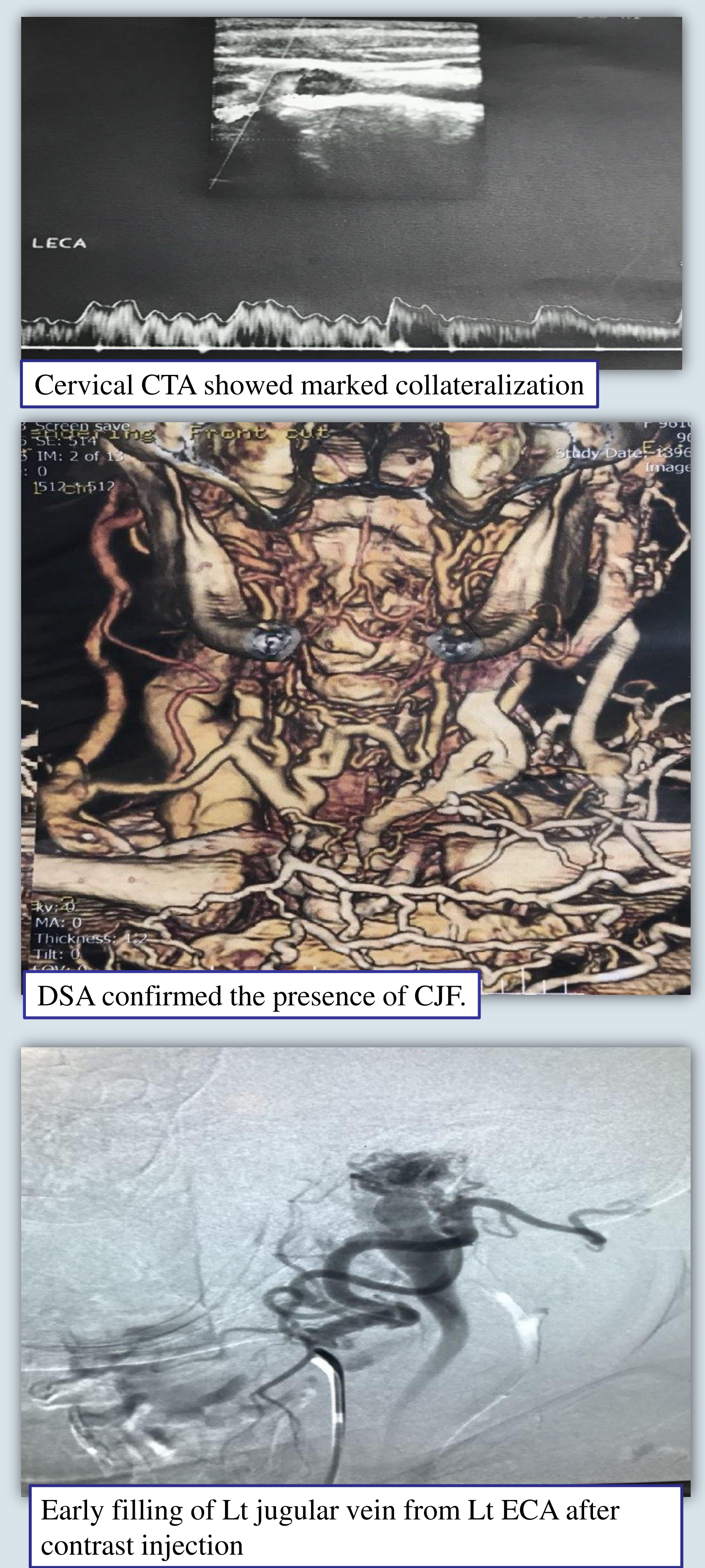

References:

-Puca AE, Pignatelli F. An Adult case of Idiopathic Internal Carotid-Internal Jugular Vein Arteriovenous Fistula. Annals of vascular surgery. 2015 May 1;29(4):842-e5.

-Caldarelli C, Biricotti M, Materazzi G, Spinelli C, Spisni R. Acquired carotid-jugular fistula: its changing history and management. ISRN Vascular Medicine. 2013 Jul 8;2013.

•Ezri T, Szmuk P, Cohen Y, Simon D, Mavor E, Katz J, Geva D. Carotid artery-internal jugular vein fistula: a complication of internal jugular vein catheterization. Journal of cardiothoracic and vascular anesthesia. 2001 Apr 1;15(2):231-2.

-Bakar B, Cekirge S, Tekkok IH. External carotid-internal jugular fistula as a late complication after carotid endarterectomy: a rare case. Cardiovascular and interventional radiology. 2011 Feb 1;34(2):53-6.

-Tan ME, Rijken M, Moll FL. Spontaneous external carotid-jugular fistula in repetitive hyperextension of the neck. Annals of vascular surgery. 2002 Dec 21;16(6):771-3. 